\title{
High-throughput and continuous flow isolation of rare circulating tumor cells and clusters in gastric cancer from human whole blood samples using electromagnetic vibration-based filtration
}

\author{
AN XIANG $^{1 *}$, MEI XUE ${ }^{2 *}$, FENGLING REN $^{3}$, LI WANG $^{1}$, \\ ZICHEN YE ${ }^{1}, \mathrm{DA} \mathrm{LI}^{1}$, QIFENG $\mathrm{JI}^{1}$, GANG $\mathrm{JI}^{4}$ and ZIFAN LU ${ }^{1}$ \\ ${ }^{1}$ State Key Laboratory of Cancer Biology, Department of Biopharmaceutics, School of Pharmacy, \\ Air Force Medical University, Xi'an, Shaanxi 710032; ${ }^{2}$ Center for Translational Medicine, \\ The First Affiliated Hospital of Xi'an Jiaotong University, Xi'an, Shaanxi 710061; ${ }^{3}$ School of Public Health, \\ Xi'an Jiaotong University Health Science Center, Xi'an, Shaanxi 710061; ${ }^{4}$ Xijing Hospital of Digestive Diseases, \\ Xijing Hospital, Air Force Medical University, Xi'an, Shaanxi 710032, P.R. China
}

Received September 17, 2019; Accepted February 19, 2020

DOI: $10.3892 /$ or.2020.7567

\begin{abstract}
Circulating tumor cells (CTCs) or CTC clusters are considered as suitable and relevant targets for liquid biopsy as they more accurately indicate cancer progression, the therapeutic effects of treatment and allows for monitoring of cancer metastasis in real-time. Among the various methods for isolating CTCs, size-based filtration is one of the most convenient methods. However, cell clogging makes the filtration process less efficient. In the present study, an electromagnetic vibration-based filtration (eVBF) device was developed that efficiently isolated rare CTCs and CTC clusters from clinical blood samples of patients with gastric cancer. Using human blood samples spiked with human gastric cancer cells, the parameters of this device such as vibrating amplitude
\end{abstract}

Correspondence to: Dr Gang Ji, Xijing Hospital of Digestive Diseases, Xijing Hospital, Air Force Medical University, Xi'an, Shaanxi 710032, P.R. China

E-mail: jigang@fmmu.edu.cn

Dr Zifan Lu, State Key Laboratory of Cancer Biology, Department of Biopharmaceutics, School of Pharmacy, Air Force Medical University, Xi'an, Shaanxi 710032, P.R. China

E-mail: luzfliuq@fmmu.edu.cn

*Contributed equally

Abbreviations: ANOVA, analysis of variance; CK, cytokeratin; CMFDA, chloromethylfluorescein diacetate; CTC, circulating tumor cell; EpCAM, epithelial cell adhesion molecule; eVBF, electromagnetic vibration-based filtration; FDA, US food and drug administration; FITC, fluorescein isothiocyanate; PBS, phosphate-buffered saline; PE, phycoerythrin; WBC, white blood cell

Key words: circulating tumor cells, circulating tumor cell clusters, rare cell isolation, electromagnetic vibration, filtration and flow rate were optimized. Putative CTCs were detected using a conventional filtration method and the eVBF device from the peripheral blood samples of patients with gastric cancer. Continuous flow isolation of CTCs was evaluated by a simulated blood flow system. The eVBF device utilized the electromagnetic force to generate a periodic vibration that prevented the cell clogging and improved the filtering efficiency. The optimized eVBF device with the high-amplitude vibration exhibited a recovery efficiency of $80-90 \%$ from whole blood samples spiked with 100 or 1,000 gastric cancer cells per $\mathrm{ml}$. Using the eVBF device, CTCs were detected in $100 \%$ of patients (10/10) with gastric cancer, and the positive detection rate of the eVBF device was $30 \%$ higher compared with the conventional filtration method. Furthermore, CTC clusters were detected in $40 \%$ (4/10) of CTC-positive patient samples, and the integrity of CTC clusters was preserved using the eVBF device. The eVBF device allowed for high-throughput (1 $\mathrm{ml} / \mathrm{min}$ ) and continuous flow isolation of CTCs without the addition of any antibodies, any chemical reagents or any pretreatment processes. Thus, the eVBF device provides an efficient tool for isolating rare CTCs and CTC clusters from patients with cancer, highlighting its potential for use in cancer diagnosis, treatment and cancer biology research.

\section{Introduction}

Cancer is the primary cause of death in the world, and the main cause of cancer-associated death is cancer metastasis, which arises following dissemination of cancer cells from the primary tumor (1). The disseminated cancer cells in blood circulation are defined as circulating tumor cells (CTCs) that are carried around the human body by the bloodstream and colonize at secondary sites forming metastatic tumors (2). It is difficult to isolate CTCs due to their rarity in the peripheral blood of patients with cancer, and only 1-100 CTCs can be detected from $1 \times 10^{9}$ hematological cells in $1 \mathrm{ml}$ human whole blood $(3,4)$. The majority of single CTCs are apoptotic owing to their loss of cell adherence and the shear forces in the 
bloodstream. Only a rare subset of aggregated CTC clusters (2-50 CTCs) are capable of surviving in the bloodstream (5). Compared to a single CTC, CTC clusters have enhanced metastatic potential, and the presence of CTC clusters is closely associated with shorter progression-free survival and overall survival in various types of metastatic cancer $(6,7)$. The isolated CTCs or CTC clusters from the liquid biopsy samples are considered to represent the molecular characteristics of the primary tumor, and culturing of CTC-derived tumors may provide valuable information on drug sensitivity and immune epitope prediction. Growing evidence has also demonstrated the clinical significance of isolated CTCs or CTC clusters in cancer prognosis, treatment response and recurrence (8-11).

Strategies for isolation and enhancement of CTCs can be classified into two major categories: Antibody-antigen affinity-based methods and isolation devices based on the physical characteristics of cancer cells (12). The only system approved by the US Food and Drug Administration (FDA) for detecting CTCs is the CellSearch ${ }^{\circledR}$ system which relies on targeting the epithelial cell adhesion molecule (EpCAM) epitope to isolate CTCs (13). The primary limitation of antibody-antigen affinity-based methods is the low detection rates, as both the expression levels and types of cancer cell biomarkers are highly dynamic and heterogeneous during cancer progression. Sample preparation of existing CTC isolation methods may also severely damage CTC clusters and fail to preserve their integrity (14). Another strategy for isolation of CTCs is based on the physical characteristics of cancer cells, such as size, density and dielectric properties (15-17). Among these methods, conventional size-based filtration has been frequently used for CTC isolation (18). The majority of CTCs are significantly larger than blood cells and thus can be isolated using a polycarbonate membrane with a pore size of $8 \mu \mathrm{m}$ (19). Conventional size-based filtration methods are more widely used in practice due to the ease of operation and low cost, and previous reports have indicated that the size-based filtration may be a more accurate method for predicting patient prognosis in certain types of cancer $(20,21)$. However, filter clogging, and a high degree of damage are the limitations of conventional filtration methods, which limit more widespread clinical use.

To overcome filter clogging and enhance the filtering efficiency, in the present study, an electromagnetic vibration-based filtration (eVBF) device was developed that could isolate rare CTCs and CTC clusters from clinical blood samples of patients with cancer. The eVBF device utilized an electromagnetic force to generate a periodic vibration that prevented filter clogging and enhanced the filtering efficiency. Based on the optimized parameters of the eVBF device, CTCs were successfully isolated from spiked human whole blood samples. The efficiency of CTC recovery with this device was $>80 \%$. The eVBF device was also used to isolate CTCs or CTC clusters in clinical blood samples of patients with gastric cancer, and the sensitivity of detection of CTCs using this method was $100 \%$. Finally, the eVBF device was also deemed suitable for continuous flow isolation of CTCs from large-volume blood samples, suggesting that it may be potentially suitable for detecting or capturing CTCs in the human body. Thus, the eVBF device represents a novel and robust technique that can efficiently isolate CTCs or CTC clusters for downstream analysis.

\section{Materials and methods}

Materials and reagents. Filters were purchased from Whatman (GE Healthcare). The polycarbonate membrane $(8 \mu \mathrm{m})$ was purchased from EMD Millipore. CK8 FITC-conjugated antibodies (cat. no. 11-9938), CK18/19 Alexa Fluor 488-conjugated antibodies (cat. nos. 53-9815 and 53-9898), EpCAM FITC-conjugated antibodies (cat. no. 11-5791) and CD45 PE-conjugated antibodies (cat. no. 12-0459) were purchased from Thermo Fisher Scientific, Inc. CellTracker Green CMFDA dye and Hoechst dye were purchased from Thermo Fisher Scientific, Inc. RPMI-1640 medium was purchased from Hyclone (GE Healthcare). FBS was purchased from Shanghai ExCell Biology, Inc. All other reagents were of analytical grade.

Design and fabrication of the eVBF device. This device was composed of an electromagnetic vibration system and a filtration system. The filtration system included a vertical filter with two chambers: A sample loading chamber with one inlet and a fluidic waste chamber with one outlet at the bottom of the filter (Fig. 1). The vertical filter was fixed on the center of the two movable cores of electromagnetic vibration. This electromagnetic vibrator adopted a half-wave rectifier and a magnetic inductor coil to produce the electromagnetic force that drove the vertical filter to vibrate cyclically. The polycarbonate membrane $(8 \mu \mathrm{m})$ was placed in the membrane holder of the vertical filter (diameter $25 \mathrm{~mm}$ ). The effluent blood cells from the fluidic waste chamber were collected in a collecting bottle via a catheter.

Cell culture and blood preparation. Human gastric cancer cells (AGS) were used to mimic CTCs. AGS cells were cultured in RPMI-1640 media supplemented with $10 \%$ FBS, maintained at $37^{\circ} \mathrm{C}$, in a humidified atmosphere with $5 \% \mathrm{CO}_{2}$ atmosphere. AGS cells were labeled with CellTracker Green CMFDA dye. Whole blood samples, obtained from healthy volunteers, were used as the spiked blood samples.

Evaluation of the eVBF device using human cancer cells spiked into whole blood samples. To evaluate the isolation efficiency of the eVBF device for CTCs, AGS cells pre-stained with CellTracker Green CMFDA dye were spiked into whole blood samples obtained from healthy individuals. Prepared blood samples were pumped into the loading chamber via a syringe pump. Spiked blood samples were injected into the upper chamber of the filter followed by filtration with a controlled amplitude or flow rate. The effluent blood cells from the fluidic waste chamber were collected in a collecting bottle. After the filtering process was complete, the filter membrane was disassembled from the vertical filter and washed with PBS. Subsequently, the washing buffer containing the CTCs was collected for further analysis.

Isolation of CTCs from clinical blood samples of patients with cancer. To evaluate the clinical applicability of the eVBF device, whole peripheral blood samples were obtained from 20 patients (11 male and 9 female patients; age range, 46-79 years) with gastric cancer from January 2017 to December 2018. The present study was approved by the Ethics 

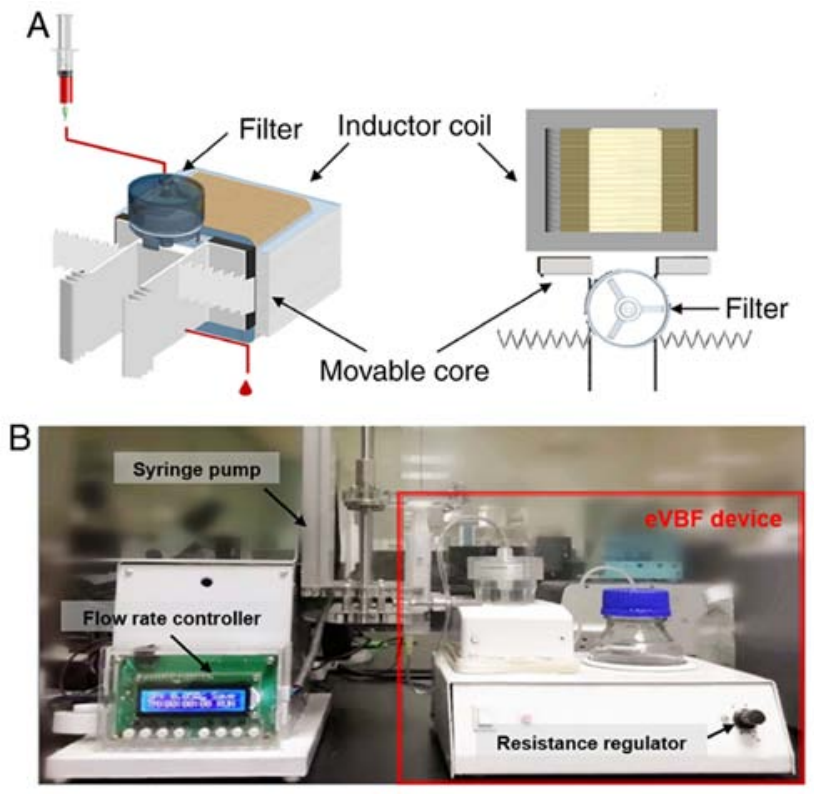

Figure 1. Schematic illustration and digital image of the eVBF device. (A) Stereo view and top view of the eVBF device with inductor coil, movable core and filter. (B) Actual digital image of the eVBF device. eVBF, electromagnetic vibration-based filtration.

Committee of Xijing Hospital of Air Force Medical University (Xi'an, China). The cells which had been isolated using the eVBF device were analyzed by immunofluorescent staining. The collected cell fractions were fixed with $4 \%$ paraformaldehyde, followed by permeabilization with $0.1 \%$ Triton X-100, and blocked with 5\% BSA. Subsequently, the cells were incubated with CK8/18/19 antibodies, EpCAM antibodies, CD45 antibodies, and cellular nuclei were counterstained with Hoechst. The imaging and counting of the isolated cells were performed using a fluorescence microscope (Olympus Corp.; original magnification, x200) and analyzed with Olympus cellSens software (version 1.6; Olympus Corp.). CTCs were identified by observing the morphology of cancer cells and the expression of biomarkers associated with cancer cells Cells which exhibited a rounded or elliptical morphology with a large nucleolus ratio, positive expression of CK8/18/19 or EpCAM (green), and negative expression of CD45 (red) were considered CTCs.

Statistical analysis. Statistical analysis was performed using GraphPad Prism version 7.0 (GraphPad Software, Inc.). Data are expressed as the mean \pm standard deviation. Differences between two groups were analyzed using a Student's t-test. Comparisons of multiple groups were analyzed using one-way analysis of variance (ANOVA) test with post hoc Student-Newman-Keuls test. $\mathrm{P}<0.05$ was considered to indicate a statistically significant difference. All model diagrams were generated using 3D Studio MAX 2016 (Autodesk, Inc.).

\section{Results}

Working principle and characterization of the eVBF device. The complete isolation system of the eVBF device is shown in Fig. 1. The device consists of a vertical filter with two chambers, and one electromagnetic vibrator with two movable cores
(Fig. 1A). The polycarbonate membrane with an $8-\mu \mathrm{m}$ pore size was placed in the membrane holder of the vertical filter. It has been previously reported that a polycarbonate membrane with an $8-\mu \mathrm{m}$ pore size is optimal for isolation of CTCs (19). In the present study, human gastric cancer AGS cells were used as a model for CTC isolation. AGS cells range in diameter from 9-20 $\mu \mathrm{m}$ which are larger than the cell size of erythrocytes or the majority of white blood cells (WBCs) as previously described $(22,23)$. During the filtering process, blood samples were filled in a syringe pump and pumped through the eVBF device under different flow rates. Furthermore, the electromagnetic vibrator of the eVBF device could drive the filter to generate periodic vibration during the filtering process, which promoted the CTCs on the membrane to vibrate and re-disperse into the cell suspension. CTCs were caught by the membrane whereas the blood cells such as erythrocytes and WBCs passed through the membrane and were collected at the bottom chamber. The vibration performance of the eVBF device was verified and the vibrating amplitude of the movable core was assessed by adjusting the resistance regulator (Fig. 1B and 2A). When the minimum current was $6 \mathrm{~mA}$, the amplitude was only $0.3 \mathrm{~mm}$. When the current was $19 \mathrm{~mA}$, the amplitude of the movable core was $0.8 \mathrm{~mm}$. When the current reached $38 \mathrm{~mA}$, the amplitude reached $1.3 \mathrm{~mm}$ (Fig. 2B).

To examine the practical isolation parameters of the eVBF device for CTC isolation, the effects of amplitude on the vibration were determined. Cell culture dishes with blood samples were used to substitute the vertical filter. It was shown that the vibration of liquid appeared in the central part of the blood suspension under low-amplitude conditions (6 mA) (Fig. 3A, D and G). As the amplitude was enhanced (19 mA), the intensity of the vibration was further strengthened. The range of vibration expanded from the center to the edge, but the vibration intensity of the surrounding area was lower than that of the central region (Fig. 3B, E and H). When the amplitude intensity was increased to the maximum value of $38 \mathrm{~mA}$, the vibration of liquid also reached its maximum. Under these conditions, a high-strength vibration formed over the entire blood suspension from the center to the edge (Fig. 3C, F and I). To further investigate the effects of vibration on the blood suspension, the blood samples were filtered using the $8-\mu \mathrm{m}$ filter membrane of the eVBF device with different vibration amplitudes. After the filtering process was finished, the intensity of the red color of the residual blood cells on the membrane was assessed. As shown in Fig. 4A, the intensity of the residual blood cells on the membrane under low-amplitude (6 mA) was $60 \%$ higher compared with the high-amplitude (38 $\mathrm{mA})$ filtration. Taken together, these data suggest that vibration with a high-amplitude $(38 \mathrm{~mA})$ can promote blood cell filtration and reduce cell clogging.

Optimization of the eVBF device using human cancer cells spiked into whole blood. To analyze the effects of vibration on the selectivity of the filter membrane for cells, $1 \times 10^{5}$ fluorescently labeled AGS cells were spiked into $10 \mathrm{ml}$ unlabeled whole blood samples from healthy donors $\left(1 \times 10^{4}\right.$ cancer cells $/ \mathrm{ml}$ ) and these samples were isolated using the eVBF device. As shown in Fig. 4B, when applying a low-amplitude vibration $(6 \mathrm{~mA}), 69.67 \pm 10.02 \%$ of the cancer cells were recovered by the membrane. As the clogging appeared and 

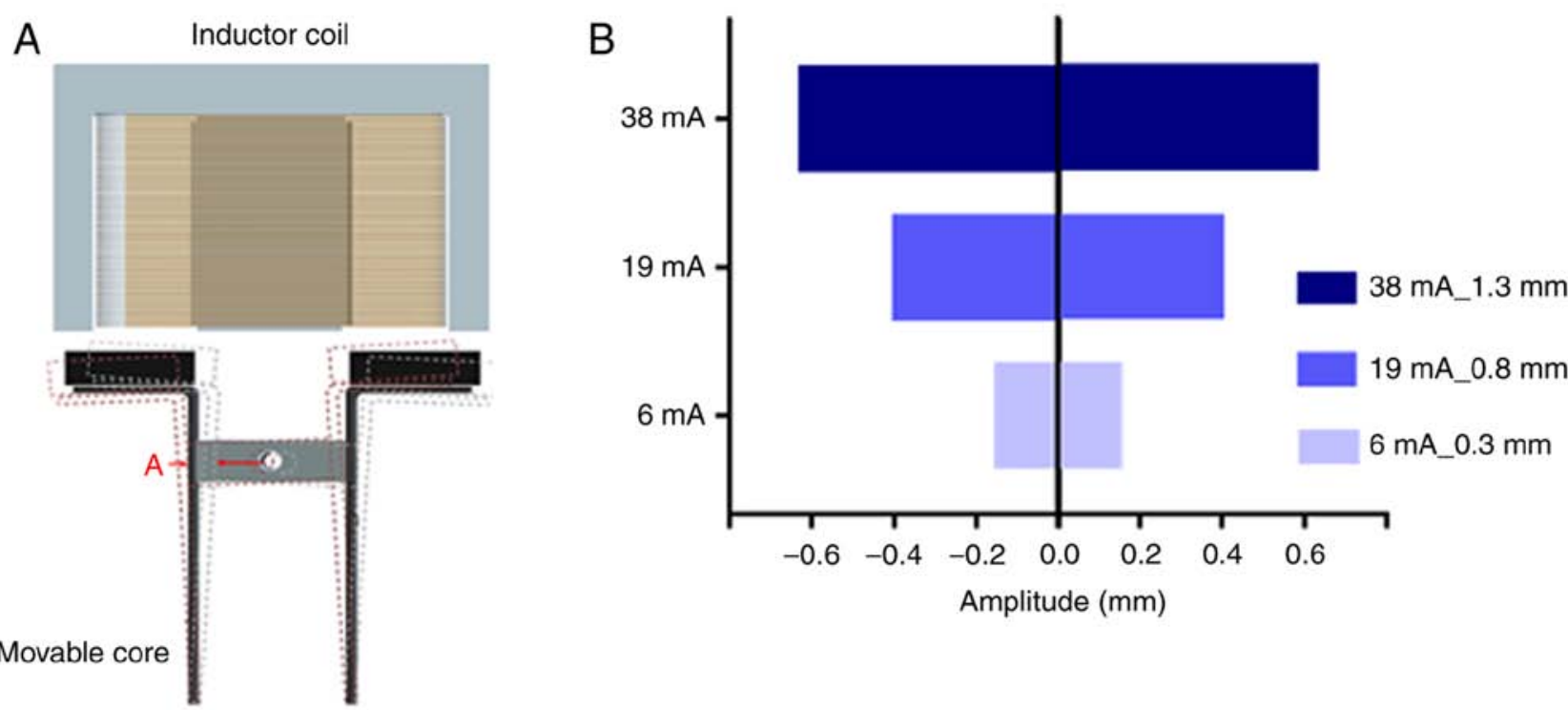

Figure 2. Identification of the optimal vibration amplitude of the eVBF device. (A) Schematic illustration of vibration of the movable core driven by the coil current. The red letter A indicates the site for measuring the amplitude, and the vertical filter is in the same horizontal line. (B) Vibration amplitude of the movable core increases with current. eVBF, electromagnetic vibration-based filtration.

A

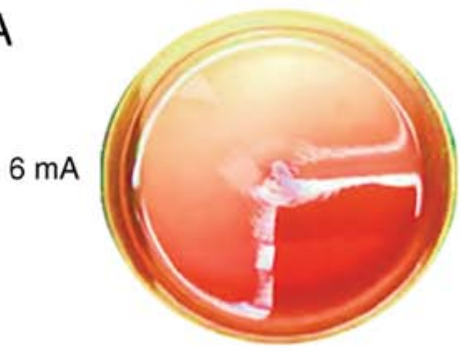

B

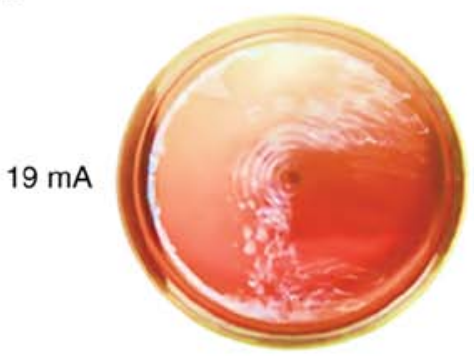

C

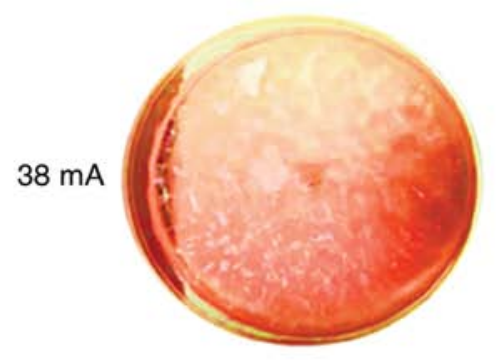

D

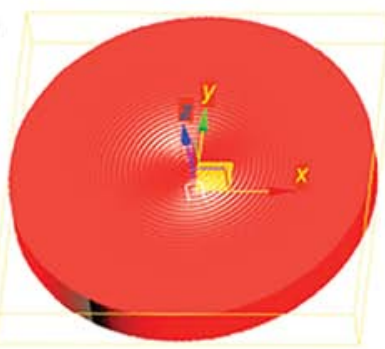

E

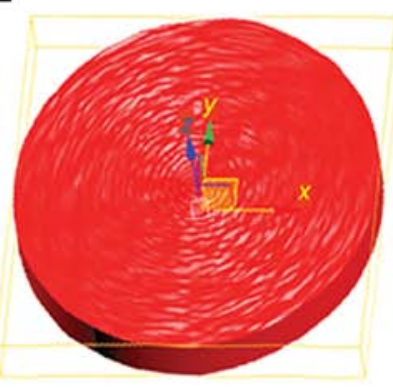

F

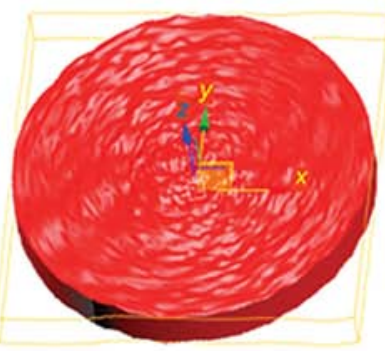

G

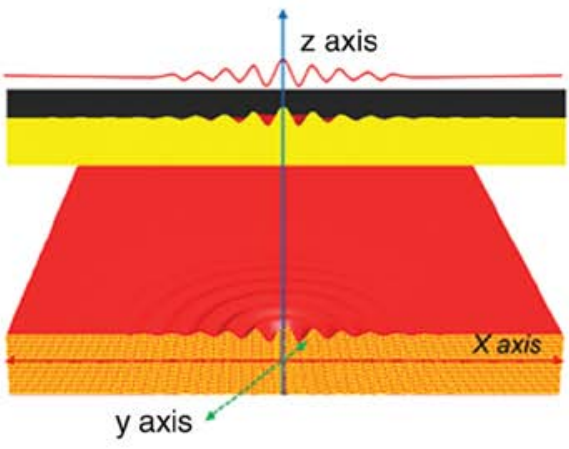

$\mathrm{H}$

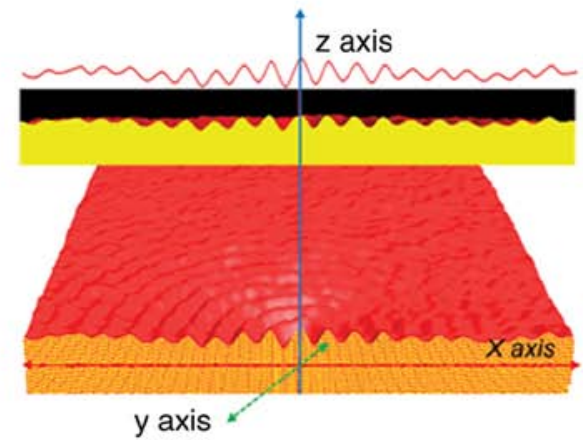

I

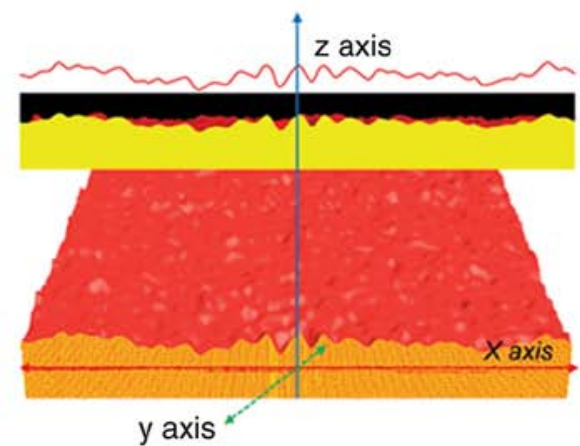

Figure 3. Characterization of the eVBF device under different vibration amplitudes. (A-C) Digital image of the vibration of the blood samples with different amplitudes (6, 19 and $38 \mathrm{~mA})$. (D-F) Schematic illustration of the vibration of the blood sample with different amplitudes (6, 19 and $38 \mathrm{~mA})$. (G-I) Sectional view of the vibration of the blood samples with different amplitudes $(6,19$ and $38 \mathrm{~mA})$. eVBF, electromagnetic vibration-based filtration. 
A
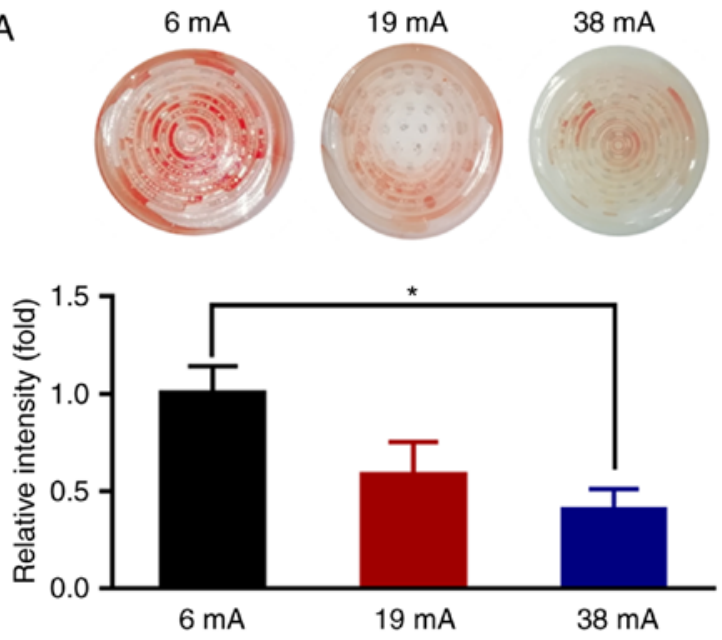

\section{B}

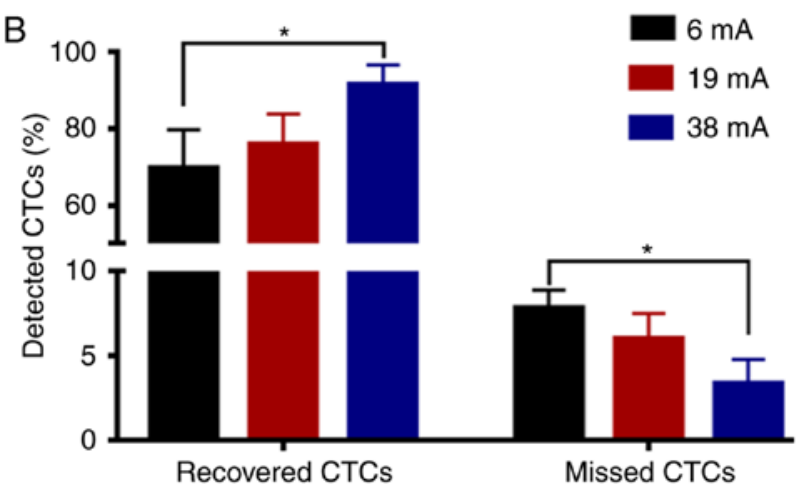

Figure 4. Optimization of the eVBF device using human gastric cancer cells spiked into whole blood samples. (A) Residual blood cells on the surface of the membrane following filtration of the blood samples using the eVBF device under different vibration amplitude conditions $(6,19$ and $38 \mathrm{~mA})$. (B) Under different vibration amplitude conditions $(6,19$ and $38 \mathrm{~mA})$, both the recovered rate of CTCs and the missed rate of CTCs were determined using fluorescence microscopy. $\mathrm{n}=3$. ${ }^{*} \mathrm{P}<0.05$. eVBF, electromagnetic vibration-based filtration; CTCs, circulating tumor cells.

progressed, $8.00 \pm 1.00 \%$ of the cancer cells became deformed and finally passed through the membrane which resulted in missed detection of CTCs. Under a medium amplitude or high-amplitude conditions (19 or $38 \mathrm{~mA}$, respectively), the recovered rate of cancer cells isolated by the filter membrane was increased from $76.00 \pm 7.76$ to $91.67 \pm 5.13 \%$, respectively, and the missed rate of cancer cells passing through the membrane was reduced from $6.00 \pm 1.50$ to $3.67 \pm 1.15 \%$, respectively (Fig. 4B). Together, these results indicate that the device may improve the isolation efficiency of CTCs and reduce the missed detection rate of CTCs.

To determine the recovery rate of the eVBF device for rare cancer cells, fluorescently labeled AGS cells were spiked into undiluted whole blood samples from healthy donors, at either $\sim 100$ cancer cells $/ \mathrm{ml}$ or $\sim 1,000$ cancer cells $/ \mathrm{ml}$. After the filtering process was complete, the residual blood cells (such as WBCs) on the filter membrane were washed and detected using immunofluorescent staining with CD45 antibodies. As shown in Fig. 5A and B, the high-amplitude (38 mA) vibration of the eVBF device improved the recovery rate of CTCs while reducing the background WBC count. Isolated experiments under high-amplitude ( $38 \mathrm{~mA}$ ) conditions were repeated 10 times. The average recovered efficiency of the
100 and 1,000 AGS cells was $80.55 \pm 13.93$ and $85.10 \pm 8.15 \%$, respectively (Fig. 5C). The effects of flow rate (throughput) for CTC isolation were further observed. The flow rate of the eVBF device was set at $0.5,1,2,3$ and $5 \mathrm{ml} / \mathrm{min}$. As shown in Fig. 5D, the flow rate of the eVBF device affected the recovery rate of CTCs. These results also indicated that as the flow rate increased, the recovery rate of CTCs decreased. Compared with a flow rate of $1 \mathrm{ml} / \mathrm{min}$, the lower flow rate of $0.5 \mathrm{ml} / \mathrm{min}$ had no significant effect on the recovery rate of CTCs. Together, these results suggest that the optimal conditions for use of the eVBF device for isolation of CTCs were a high-amplitude vibration $(38 \mathrm{~mA})$ and a high throughput $(1 \mathrm{ml} / \mathrm{min})$.

Isolation of CTCs and CTC clusters from blood samples of gastric cancer patients using the eVBF device. To evaluate the clinical applicability of the eVBF device, 5-ml blood samples were collected from 10 patients with gastric cancer. The collected blood samples were divided into two equal parts: One tube for conventional filtration and the other tube for filtration using the eVBF device (Fig. 6A). The isolated cells were identified using immunofluorescent staining. Cells that showed a round or elliptical morphology with a large nucleolus ratio, positive expression of CK8/18/19 or EpCAM (green), and negative expression of CD45 (red) were considered CTCs. The clinical information and CTC count for each patient are presented in Table I. CTCs were detected in 10 out of 10 (100\%) patient blood samples using the eVBF device, and 7 out of $10(70 \%)$ of the patient blood samples using a conventional filtration method. (Fig. 6B and C). The enumeration of isolated CTCs using the eVBF device ranged between 5-22 tumor cells in $2.5 \mathrm{ml}$ of blood, and the enumeration of isolated CTCs using the conventional filtration method ranged between 1-13 tumor cells in $2.5 \mathrm{ml}$ of blood. Representative immunofluorescent staining images of single CTCs are shown in Fig. 6D. To evaluate the capability of the eVBF device for isolation of CTC clusters, 5-ml blood samples were collected from another 10 patients with gastric cancer. CTC clusters were identified in 4 of $10(40 \%)$ patients with gastric cancer. As shown in Fig. 6D, the integrity of CTC clusters was preserved using this device. Therefore, compared with conventional filtration methods, the eVBF device more effectively isolated CTCs or CTC clusters from whole blood samples of patients with gastric cancer compared with conventional filtration methods.

Continuous flow isolation of CTCs from whole blood using the eVBF device. Owing to the extremely low concentration of CTCs in the peripheral bloodstream, isolated CTCs within a limited volume $(5-10 \mathrm{ml})$ of blood samples may reduce the accuracy of detection (24). Methods for continuous flow isolation of CTCs in vivo have been demonstrated that may overcome this limitation $(25,26)$. To explore the capability of the eVBF device to isolate CTCs from large-volume blood samples ( $>100 \mathrm{ml})$, a peristaltic pump was used to control the flow rate at $1 \mathrm{ml} / \mathrm{min}$. This peristaltic pump also pumped the blood flow through the eVBF device (Fig. 7A). Subsequently, a syringe pump was used to continuously inject $1 \times 10^{4}$ fluorescently labeled AGS cells into the blood flow (10 ml undiluted whole blood samples) for $120 \mathrm{~min}$ (equal to total $120 \mathrm{ml}$ blood samples and $\sim 80$ cancer cells $/ \mathrm{ml}$ ) to simulate the CTCs in the bloodstream. As shown in Fig. 7B, the average recovery rate 

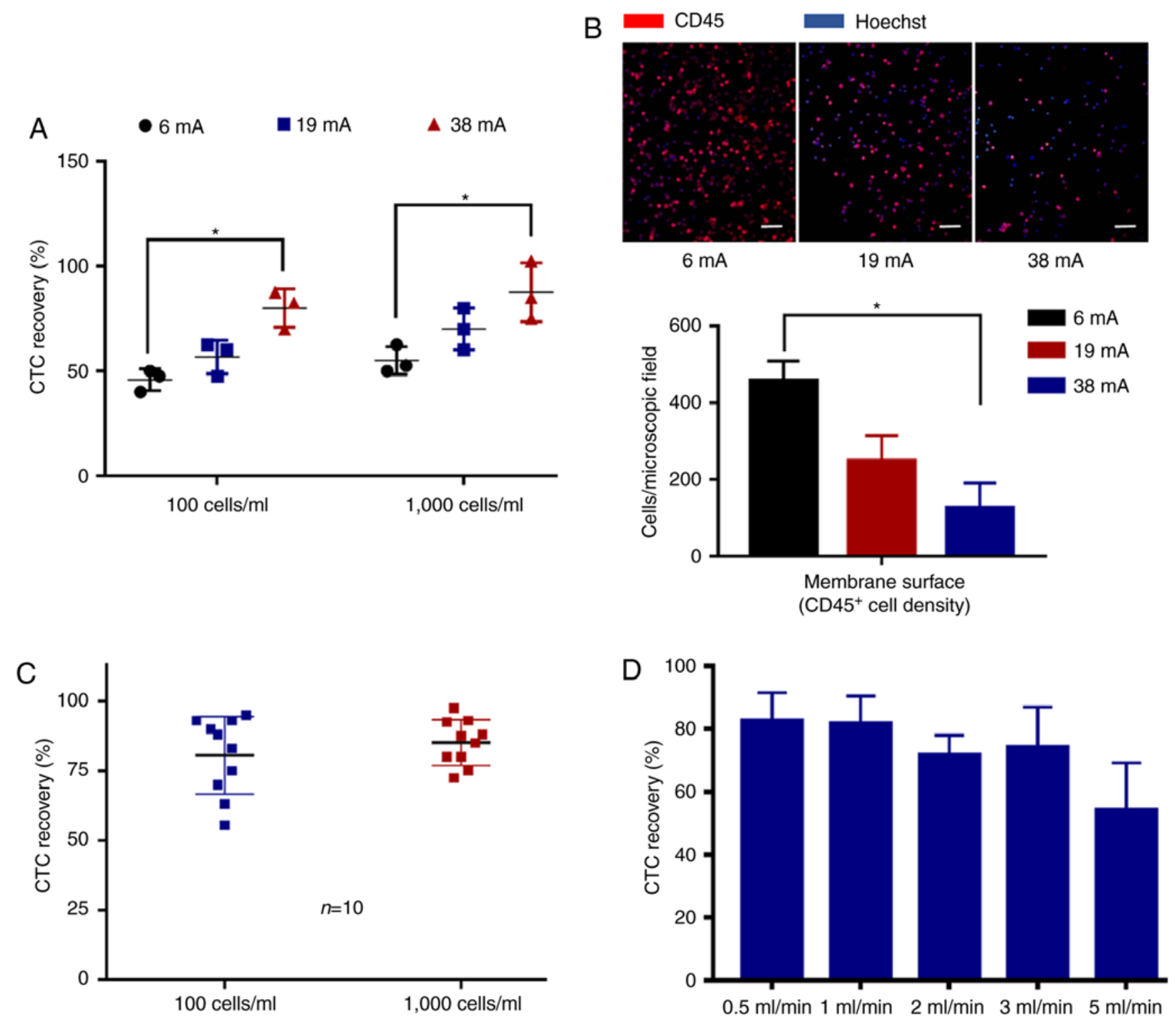

Figure 5. Efficiency of CTCs recovery using the eVBF device under different conditions. (A) Efficiency of CTCs recovery using the eVBF device under different vibration amplitude conditions $(6,19$ and $38 \mathrm{~mA})$. $\mathrm{n}=3 .{ }^{*} \mathrm{P}<0.05$. (B) Immunofluorescent analysis of background blood cells under different vibration amplitude conditions (6,19 and $38 \mathrm{~mA}$ ). Scale bar, $100 \mu \mathrm{m} . \mathrm{n}=3$. ${ }^{*} \mathrm{P}<0.05$. (C) Efficiency of CTCs recovery under high-amplitude ( $\left.38 \mathrm{~mA}\right)$ conditions. Measurements represent the average values of 10 independent experiments. (D) CTCs recovery efficiency of the eVBF device under different flow rates (throughput, $0.5,1,2$, 3 and $5 \mathrm{ml} / \mathrm{min}$ ). eVBF, electromagnetic vibration-based filtration; CTCs, circulating tumor cells.

of CTCs was $92.50 \pm 6.32 \%$. When the filtering process was completed, the average residual rates of CTCs in the bloodstream was $2.93 \pm 0.30 \%$ (Fig. 7B). The other $5 \%$ of CTCs may have remained in the pipeline or were lost during the filtering process. Taken together, these results suggest that the eVBF device is suitable for isolation of CTCs in large volumes of blood samples due to effective reduction of cell clogging.

To simulate the complicated blood circuit of humans, the blood flow system described above was expanded upon by adding another peristaltic pump to simulate blood reflux. Subsequently, $5 \times 10^{5}$ fluorescently labeled AGS cells were injected into $10 \mathrm{ml}$ of undiluted whole blood samples. One part of this mixture was passed through the eVBF device at a flow rate of $1 \mathrm{ml} / \mathrm{min}$, whereas the other part of the blood flow was retransferred without passing through the $\mathrm{EBF}$ device at a flow rate of 1,2 or $3 \mathrm{ml} / \mathrm{min}$ (Fig. 7C). The concentrations of residual CTCs in the blood flow system were measured at different time points (every $5 \mathrm{~min}$ for a total of $30 \mathrm{~min}$ ) to evaluate the ability of the eVBF device to isolate CTCs. As the reflux rate increased, the blood flow pumped into the eVBF device was reduced, and more CTCs remained in the blood flow that was unfiltered by eVBF device. As the filtering time increased, the residual number of CTCs in the blood flow decreased gradually (Fig. 7D-F). Furthermore, the actual reduction of CTCs in the blood flow was lower than the theoretical value (red curve) at different filtering time points.

Under different filtering time points $(t)$, the calculation of the theoretical value of the residual concentration of CTCs in the blood flow $\left(C_{t}\right)$ is described by equation $1: C^{t}=C^{0}\left[\mathrm{~V} /(1+V]^{N}\right.$, where $\mathrm{C}_{0}$ represents the initial concentration of CTCs in the blood flow. The flow rate of the blood loop through the eVBF device was $1 \mathrm{ml} / \mathrm{min}$, and the flow rate of the blood loop without the $\mathrm{eVBF}$ device was $\mathrm{V} \mathrm{ml} / \mathrm{min}$. The total velocity of 
A

Rinse PBS
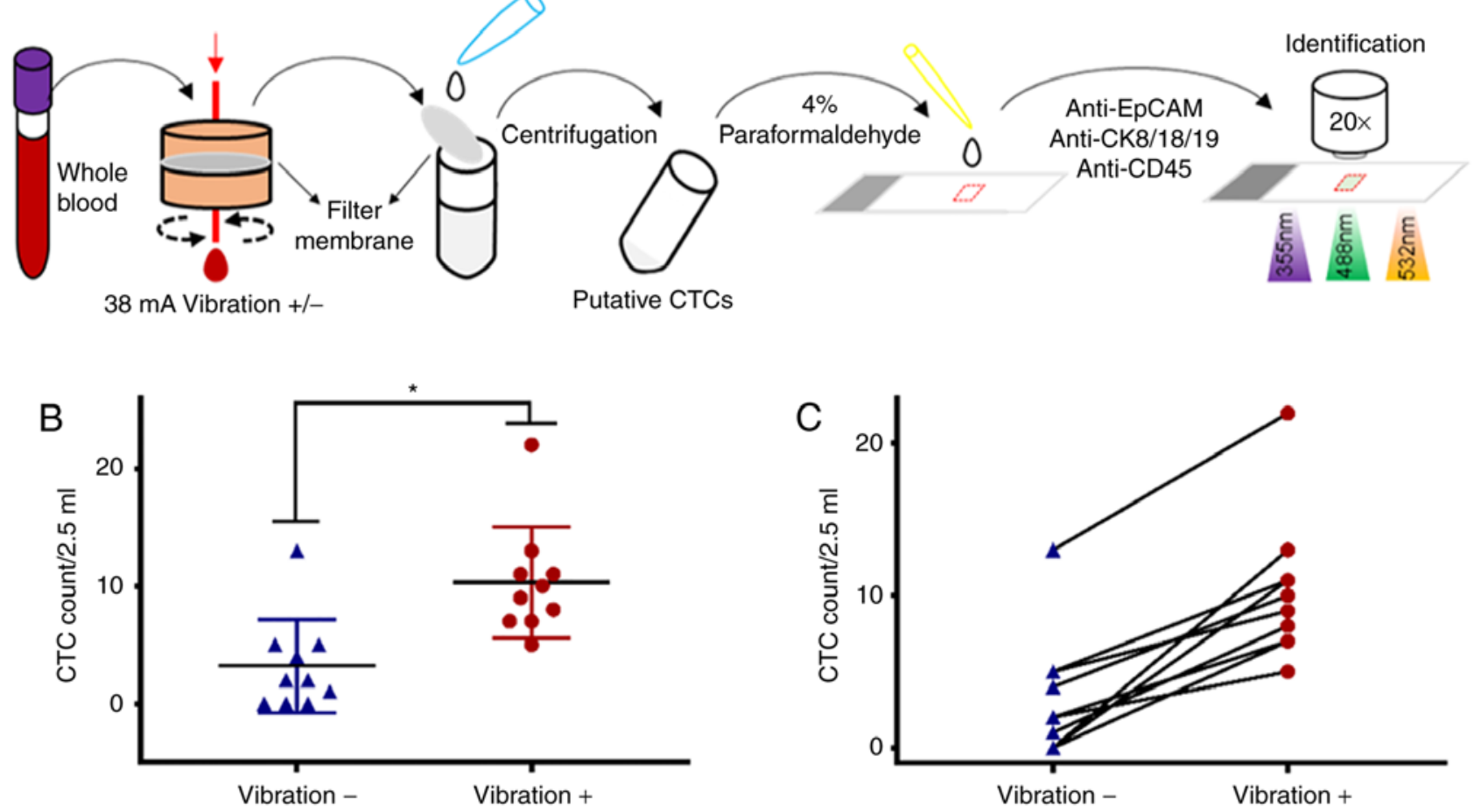

D

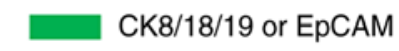

CD45

Hoechst
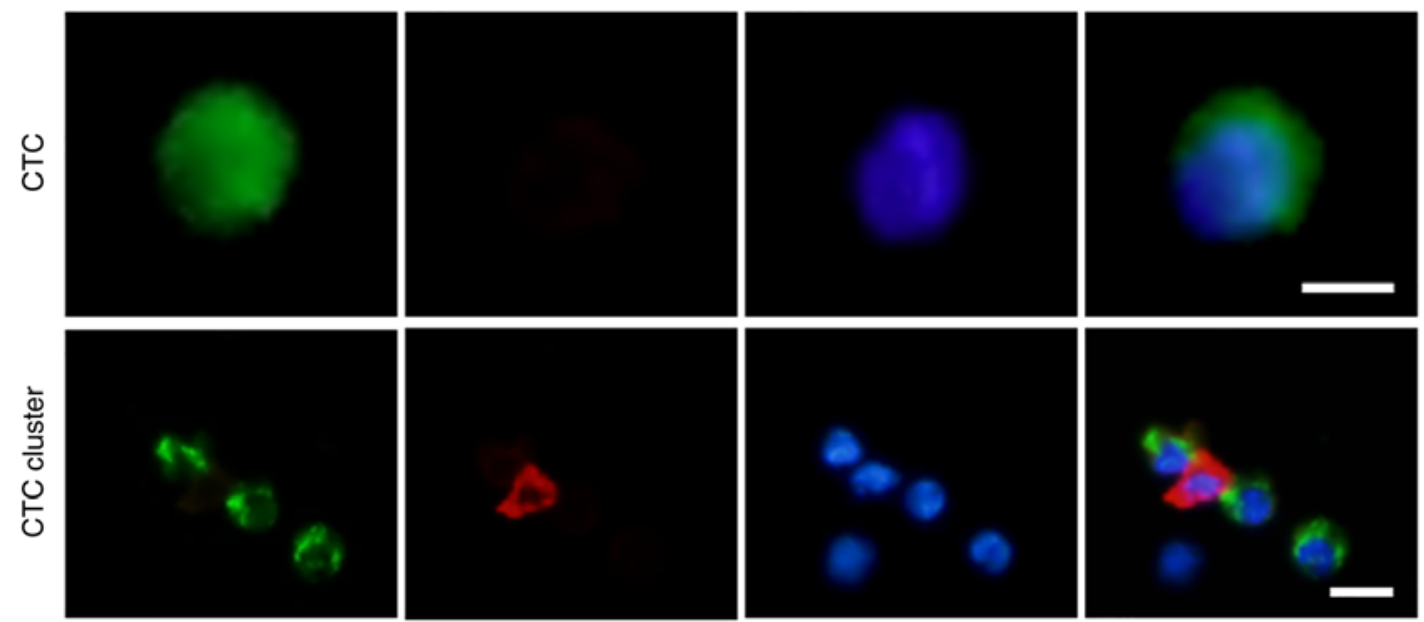

Figure 6. Isolation of CTCs from clinical blood samples of patients with gastric cancer using the eVBF device. (A) Workflow of CTCs isolation from clinical samples using the eVBF device. (B and C) Comparisons of the enumeration of CTCs between the conventional filtration method and the eVBF device. $\mathrm{n}=10$. ${ }^{*} \mathrm{P}<0.05$. (D) Immunofluorescent images of the identified CTCs or CTC clusters from clinical blood samples of patients with gastric cancer. Cells which showed a round or elliptical morphology with a large nucleolus ratio, positive expression of CK8/18/19 or epithelial cell adhesion molecule (green), and negative expression of CD45 (red) were considered CTCs. Scale bar, $10 \mu \mathrm{m}$. eVBF, electromagnetic vibration-based filtration; CTCs, circulating tumor cells.

blood circulation was $(\mathrm{V}+1) \mathrm{ml} / \mathrm{min}$. Theoretically, all CTCs passing through the eVBF device may be intercepted by the filter membrane. When the filtering time is $t \mathrm{~min}$, the actual filtration period $(\mathrm{N})$ is described by equation $2: \mathrm{N}=\mathrm{t}(\mathrm{V}+1) / 10$. Thus, the theoretical value of the residual concentration of CTCs in the blood flow $\left(C_{t}\right)$ could be represented as equation 1 and equation 2 . When the flow rate of blood reflux increased, the residual amount of CTCs in the blood flow also increased (Fig. 7D-F). Together, these results suggest that this device significantly reduced the total content of CTCs in the bloodstream. However, to achieve the desired isolated rate of CTCs, the filtering time, the filter membrane area or the blood samples passed through the eVBF device should be increased suitably.

\section{Discussion}

Conventional size-based filtration is a tumor biomarker-independent method for isolation of CTCs, and the advantages of this technique mainly include simple structure, convenient operation and low cost (27). However, the primary challenge of conventional filtration methods is cell clogging, which results in a low enrichment efficiency of CTCs. Severe cell clogging can form on the surface of the filter membrane when 
Table I. Clinical information of the 10 patients with gastric cancer and the numbers of CTCs detected.

CTC count

\begin{tabular}{cclllrr} 
Patient ID & Age (years) & Sex & Clinical diagnosis & Clinical treatment & Conventional filtration & eVBF device \\
\hline 1 & 67 & Male & Gastric cancer & After operation & 4 & 10 \\
2 & 60 & Female & Gastric cancer & Before operation & 5 & 11 \\
3 & 46 & Male & Gastric cancer & After operation & 13 & 22 \\
4 & 79 & Male & Gastric cancer & Before operation & 5 & 9 \\
5 & 79 & Male & Gastric cancer & After operation & 0 & 1 \\
6 & 65 & Female & Gastric cancer & After operation & 0 & 13 \\
7 & 51 & Male & Gastric cancer & After operation & 2 & 7 \\
8 & 52 & Male & Gastric cancer & Before operation & 2 & 7 \\
9 & 54 & Male & Gastric cancer & After operation & & 7 \\
10 & 51 & Female & Gastric cancer & After operation & 0 &
\end{tabular}

CTCs, circulating tumor cells; eVBF, electromagnetic vibration-based filtration.
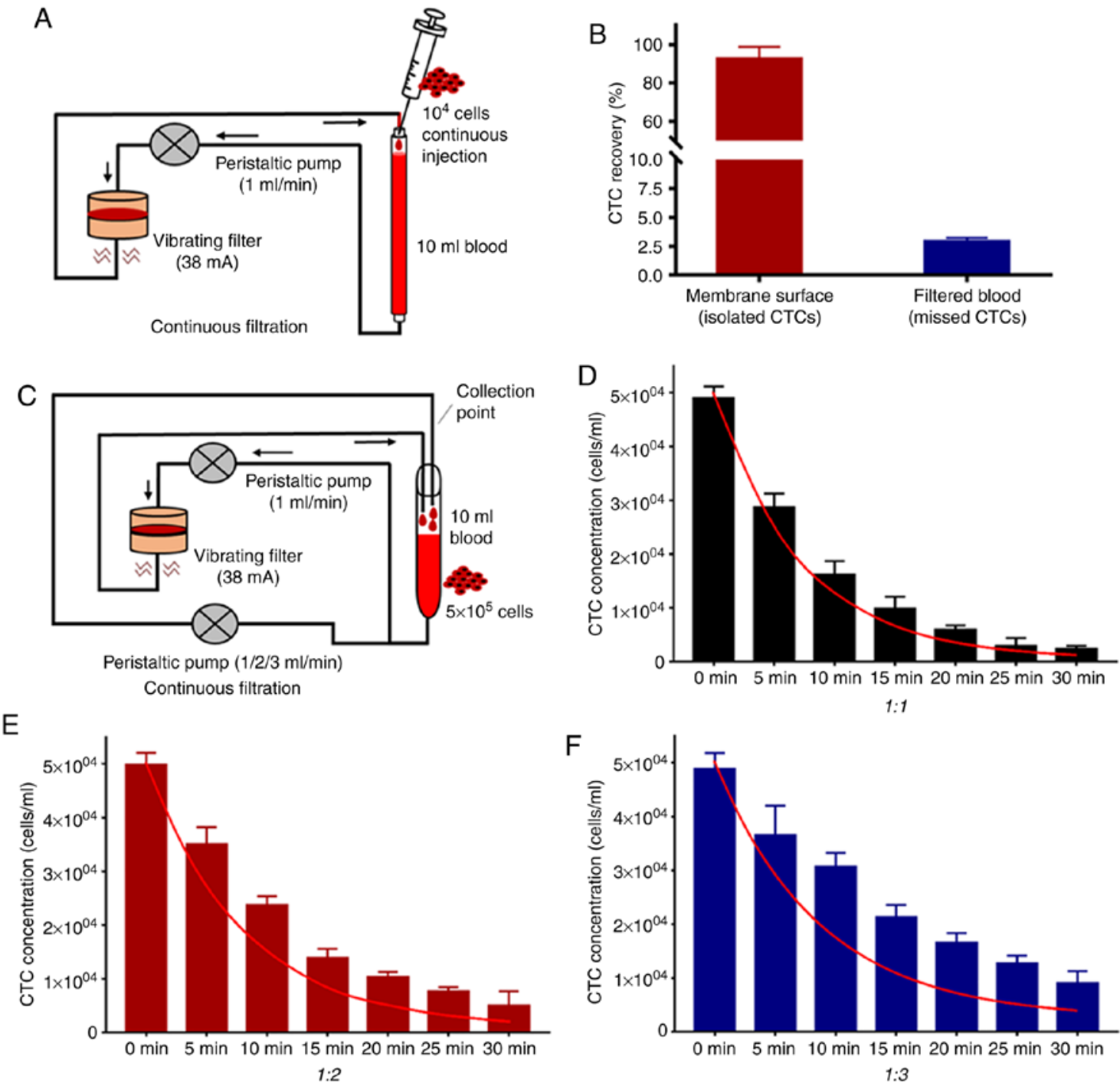

Figure 7. Continuous flow isolation of CTCs using the eVBF device. (A) Schema for simulating continuous flow isolation of CTCs using the eVBF device. (B) Recovery rate and residual rate of continuous isolation of CTCs for $2 \mathrm{~h}$. (C) Schema for simulating human blood flow for isolation of CTCs using the eVBF device. (D-F) Residual concentrations of CTCs in the blood flow system at different filtering time points. The ratio of the flow velocity of the filter circuit to that of the unfiltered circuit was 1:1, 1:2 or 1:3. The red curve represents the theoretical values of residual concentrations of CTCs in the blood flow. eVBF, electromagnetic vibration-based filtration; CTCs, circulating tumor cells. 
A
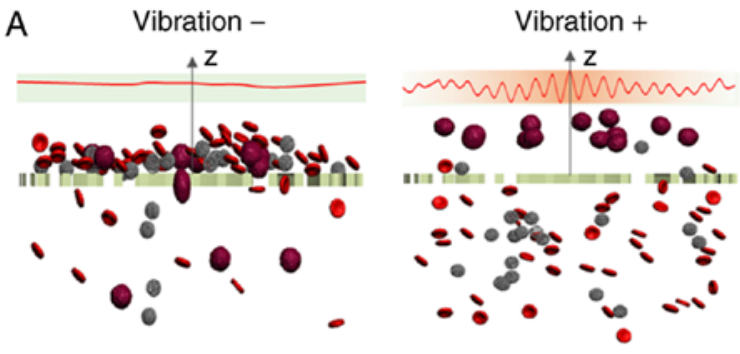

Erythrocyte Leukocyte
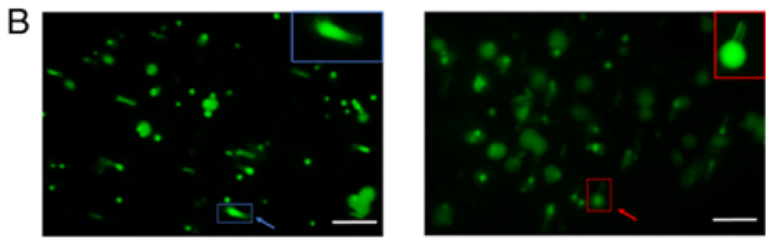

Figure 8. Schematic illustration of the working principles of conventiona filtration methods and the eVBF device for CTCs isolation. (A) Sectional view of the filter during the filtering process with and without vibration (B) Fluorescence images of labeled AGS cancer cells captured by the filter membrane with and without vibration. Blue colors indicate deformed cancer cells which passed through the membrane pores without vibration. Red colors indicate the cancer cells which were not deformed and captured by the membrane with vibration. Scale bar, $50 \mu \mathrm{m}$. eVBF, electromagnetic vibration-based filtration; CTCs, circulating tumor cells.

using conventional filtration methods, which may cause cancer cells to become deformed and pass through the membrane pores, resulting in a lowering of the detection rate of CTCs. To eliminate cell deformation, and to improve enrichment efficiency of CTCs, the conventional filtration method usually requires formaldehyde to fix the blood samples, but the fixation step can affect the downstream analyses of CTCs, such as cellular and molecular assays (28). The majority of improved filtration methods for CTC isolation are primarily modifications of traditional filter membranes to overcome the problems of low recovery and cell fixation (27-29). However, these methods require microfabrication and special materials, and the preparation processes of membranes are complex and expensive. In the present study, a simple and inexpensive eVBF device was developed for isolation of CTCs with traditional polycarbonate membranes without the use of any antibodies, chemical regents or any pretreatment processes. Periodic vibration generated by the eVBF device improved the enrichment efficiency of conventional filtration methods by preventing cell clogging. Due to the prevention of cell clogging, CTCs were not deformed and thus captured by the membrane (Fig. 8). The only FDA-approved technique for CTC isolation is the CellSearch ${ }^{\circledR}$ system which relies on the detection of the expression of the cell surface epithelial marker EpCAM to isolate CTCs from whole blood. However, the expression of EpCAM is often downregulated or absent in malignant epithelial CTCs undergoing epithelial-to-mesenchymal transition or in malignancies of non-epithelial origin (30). Compared with the FDA-approved CellSearch ${ }^{\circledR}$ system, the eVBF device is not dependent on the expression of cell surface markers. Thus, the eVBF device is able to capture sub-populations of CTCs with malignant potential that may be more suitable for phenotype identification and downstream analysis.
In patients with gastric cancer, the eVBF device exhibited $30 \%$ better clinical sensitivity compared with conventional filtration methods. The enumeration of isolated CTCs using the eVBF device (2-8.8 CTCs per $\mathrm{ml}$ ) was significantly higher compared with conventional filtration methods (0.4-5.2 CTCs per $\mathrm{ml}$ ). These results indicate that the conventional filtration method leads to the loss of CTCs, and the eVBF device could reduce this loss rate. The presence and high numbers of CTC clusters have been demonstrated, and is associated with a poor prognosis in patients with gastric cancer. CTC clusters are considered to possess a higher metastatic potential compared with single CTCs (31). Therefore, CTC clusters may also serve as biomarkers for indicating cancer prognosis and monitoring cancer metastasis. CTC clusters were isolated and identified using the eVBF device in $40 \%$ (4/10) of patients with gastric cancer. The majority of existing technologies for isolation of CTCs are designed to isolate single CTCs and the pretreatment procedures of these methods may affect the integrity of CTC clusters (14). However, the eVBF device can isolate CTC clusters from the whole blood of cancer patients and the intact CTC clusters isolated by the eVBF device can be used for downstream studies, such as molecular characterization and functional analysis.

CTCs are extremely rare in the peripheral bloodstream of humans. It is important to acquire the maximum number of CTCs from a blood sample for an accurate representation of the CTCs present (24). In in vitro methods for detection of CTCs, the volume of blood samples used for detection is limited to $5-10 \mathrm{ml}$, which may result in sampling bias and reduce the accuracy of the detection methods. To avoid sampling errors, CTCs should be isolated from considerably larger volumes of blood samples $(32,33)$. In the present study, it was demonstrated that the eVBF device could isolate CTCs from a large volume of blood sample (high-throughput $1 \mathrm{ml} / \mathrm{min}$, $>100 \mathrm{ml}$ blood sample), and the recovery rate was maintained at $\sim 90 \%$. The currently available in vivo enrichment strategies for CTC isolation have potential for monitoring the dynamics of CTCs continuously and preventing cancer metastasis in humans. However, the human blood circulatory system is very complicated, such as the differences in blood flow between the arteries and veins, the differences of blood flow in different organs, and capillary barriers, which may affect the spatial and temporal distributions of CTCs within the blood circulation of humans (34). Therefore, in vivo CTC isolation technologies are still experimental, and it is difficult to translate these technologies from the laboratory to clinical practice. These factors can also affect the clinical application of the eVBF device for continuous flow isolation of CTCs in vivo. Detailed animal studies or preclinical studies on the eVBF device application in vivo are thus required.

In summary, a simple, inexpensive and robust eVBF device for isolation of rare CTCs and CTC clusters was developed. Periodic vibration generated by the eVBF device improves conventional filtration methods for CTC isolation by preventing filter clogging. In addition, this device enables high-throughput and continuous flow isolation of CTCs without the need for antibodies, chemical regents or any pretreatment processes. Further optimization and characterization of the eVBF device is required; however, the device possesses clinical potential for real-time monitoring of cancer, which may improve patient 
outcomes and assist in improving our understanding of CTC biology.

\section{Acknowledgements}

Not applicable.

\section{Funding}

This research was funded by the National Key R\&D Program of China (no. 2017YFC1308600), the National Natural Science Foundation of China (no. 81301513), the Natural Science Foundation of Shaanxi Province of China (no. 2018JM7032) and the Key Projects of State Key Laboratory of Cancer Biology (no. CBSKL2014Z06).

\section{Availability of data and materials}

The datasets used and/or analyzed during the present study are available from the corresponding author on reasonable request.

\section{Authors' contributions}

AX, MX, GJ and ZL designed this study. AX, MX, FR, LW, ZY, DL and QJ performed the experiments and collected and analyzed the data. AX, MX and ZL wrote and revised the manuscript. All authors read and approved the final manuscript and agree to be accountable for all aspects of the research in ensuring that the accuracy or integrity of any part of the work are appropriately investigated and resolved.

\section{Ethics approval and consent to participate}

This study was approved by the Ethics Committee of Xijing Hospital of Air Force Medical University (Xi'an, China).

\section{Patient consent for publication}

Informed consent was obtained from each patient or healthy volunteer before blood sample collection.

\section{Competing interests}

The authors declare that they have no competing interests.

\section{References}

1. Siegel RL, Miller KD and Jemal A: Cancer statistics, 2019. CA Cancer J Clin 69: 7-34, 2019.

2. Ortiz V and Yu M: Analyzing circulating tumor cells one at a time. Trends Cell Biol 28: 764-775, 2018.

3. Wang S, Liu K, Liu J, Yu ZT, Xu X, Zhao L, Lee T, Lee EK, Reiss J, Lee YK, et al: Highly efficient capture of circulating tumor cells by using nanostructured silicon substrates with integrated chaotic micromixers. Angew Chem Int Ed Engl 50: 3084-3088, 2011.

4. Alix-Panabières $\mathrm{C}$ and Pantel $\mathrm{K}$ : Challenges in circulating tumour cell research. Nat Rev Cancer 14: 623-631, 2014.

5. Aceto N, Bardia A, Miyamoto DT, Donaldson MC, Wittner BS, Spencer JA, Yu M, Pely A, Engstrom A, Zhu H, et al: Circulating tumor cell clusters are oligoclonal precursors of breast cancer metastasis. Cell 158: 1110-1122, 2014.
6. Hou JM, Krebs MG, Lancashire L, Sloane R, Backen A, Swain RK, Priest LJ, Greystoke A, Zhou C, Morris K, et al: Clinical significance and molecular characteristics of circulating tumor cells and circulating tumor microemboli in patients with small-cell lung cancer. J Clin Oncol 30: 525-532, 2012.

7. Giuliano M, Shaikh A, Lo HC, Arpino G, De Placido S, Zhang XH,Cristofanilli M, Schiff R and Trivedi MV: Perspective on circulating tumor cell clusters: Why it takes a village to metastasize. Cancer Res 78: 845-852, 2018.

8. Chikaishi Y, Yoneda K, Ohnaga $\mathrm{T}$ and Tanaka F: EpCAM-independent capture of circulating tumor cells with a 'universal CTC-chip'. Oncol Rep 37: 77-82, 2017.

9. Abdallah EA, Braun AC, Flores BCTCP, Senda L, Urvanegia AC, Calsavara V, Fonseca de Jesus VH, Almeida MFA, Begnami MD, Coimbra FJF, et al: The potential clinical implications of circulating tumor cells and circulating tumor microemboli in gastric cancer. Oncologist 24: e854-e863, 2019.

10. Chen JY, Tsai WS, Shao HJ, Wu JC, Lai JM, Lu SH, Hung TF, Yang CT, Wu LC, Chen JS, et al: Sensitive and specific biomimetic lipid coated microfluidics to isolate viable circulating tumor cells and microemboli for cancer detection. PLoS One 11: e0149633, 2016.

11. Warkiani ME, Khoo BL, Wu L, Tay AK, Bhagat AA, Han J and Lim CT: Ultra-fast, label-free isolation of circulating tumor cells from blood using spiral microfluidics. Nat Protoc 11: 134-148, 2016.

12. Kaiser J: Medicine. Cancer's circulation problem. Science 327: 1072-1074, 2010

13. Yu M, Bardia A, Wittner BS, Stott SL, Smas ME, Ting DT, Isakoff SJ, Ciciliano JC, Wells MN, Shah AM, et al: Circulating breast tumor cells exhibit dynamic changes in epithelial and mesenchymal composition. Science 339: 580-584, 2013.

14. Sarioglu AF, Aceto N, Kojic N, Donaldson MC,Zeinali M,Hamza B, Engstrom A, Zhu H, Sundaresan TK, Miyamoto DT, et al: A microfluidic device for label-free, physical capture of circulating tumor cell clusters. Nat Methods 12: 685-691, 2015.

15. Warkiani ME, Guan G, Luan KB, Lee WC, Bhagat AA, Chaudhuri PK, Tan DS, Lim WT, Lee SC, Chen PC, et al: Slanted spiral microfluidics for the ultra-fast, label-free isolation of circulating tumor cells. Lab Chip 14: 128-137, 2014.

16. Li M and Anand RK: High-throughput selective capture of single circulating tumor cells by dielectrophoresis at a wireless electrode array. J Am Chem Soc 139: 8950-8959, 2017.

17. Wang K, Zhou L, Zhao S, Cheng Z, Qiu S, Lu Y, Wu Z, Abdel Wahab AHA, Mao $\mathrm{H}$ and Zhao J: A microfluidic platform for high-purity separating circulating tumor cells at the single-cell level. Talanta 200: 169-176, 2019.

18. Vona G, Sabile A, Louha M, Sitruk V, Romana S, Schütze K, Capron F, Franco D, Pazzagli M, Vekemans M, et al: Isolation by size of epithelial tumor cells: A new method for the immunomorphological and molecular characterization of circulatingtumor cells. Am J Pathol 156: 57-63, 2000.

19. Hao SJ, Wan Y, Xia YQ, Zou X and Zheng SY: Size-based separation methods of circulating tumor cells. Adv Drug Deliv Rev 125: 3-20, 2018

20. Farace F, Massard C, Vimond N, Drusch F, Jacques N, Billiot F, Laplanche A, Chauchereau A, Lacroix L, Planchard D, et al: A direct comparison of CellSearch and ISET for circulating tumour-cell detection in patients with metastatic carcinomas. $\mathrm{Br}$ J Cancer 105: 847-853, 2011.

21. De Giorgi V, Pinzani P, Salvianti F, Panelos J, Paglierani M, Janowska A, Grazzini M, Wechsler J, Orlando C, Santucci M, et al: Application of a filtration- and isolation-by-size technique for the detection of circulating tumor cells in cutaneous melanoma. J Invest Dermatol 130: 2440-2447, 2010.

22. Coumans FA, van Dalum G, Beck M and Terstappen LW: Filter characteristics influencing circulating tumor cell enrichment from whole blood. PLoS One 8: e61770, 2013.

23. Shapiro HM, Schildkraut ER, Curbelo R, Laird CW, Turner B and Hirschfeld T: Combined blood cell counting and classification with fluorochrome stains and flow instrumentation. J Histochem Cytochem 24: 396-401, 1976.

24. Li P, Mao Z, Peng Z, Zhou L, Chen Y, Huang PH, Truica CI, Drabick JJ, El-Deiry WS, Dao M, et al: Acoustic separation of circulating tumor cells. Proc Natl Acad Sci USA 112: 4970-4975, 2015.

25. Galanzha EI, Menyaev YA, Yadem AC, Sarimollaoglu M, Juratli MA, Nedosekin DA, Foster SR, Jamshidi-Parsian A, Siegel ER, Makhoul I, et al: In vivo liquid biopsy using cytophone platform for photoacoustic detection of circulating tumor cells in patients with melanoma. Sci Transl Med 11: pii: eaat5857, 2019. 
26. Saucedo-Zeni N, Mewes S, Niestroj R, Gasiorowski L, Murawa D, Nowaczyk P, Tomasi T, Weber E, Dworacki G, Morgenthaler NG, et al: A novel method for the in vivo isolation of circulating tumor cells from peripheral blood of cancer patients using a functionalized and structured medical wire. Int J Oncol 41: 1241-1250, 2012.

27. Zheng S, Lin H, Liu JQ, Balic M, Datar R, Cote RJ and Tai YC: Membrane microfilter device for selective capture, electrolysis and genomic analysis of human circulating tumor cells J Chromatogr A 1162: 154-161, 2007.

28. Zheng S, Lin HK, Lu B, Williams A, Datar R, Cote RJ and Tai YC: 3D microfilter device for viable circulating tumor cell (CTC) enrichment from blood. Biomed Microdevices 13: 203-213, 2011.

29. Lin E, Cao T, Nagrath S and King MR: Circulating tumor cells: Diagnostic and therapeutic applications. Annu Rev Biomed Eng 20: 329-352, 2018.

30. Iliescu FS, Poenar DP, Yu F, Ni M, Chan KH, Cima I, Taylor HK, Cima I and Iliescu C: Recent advances in microfluidic methods in cancer liquid biopsy. Biomicrofluidics 13: 041503, 2019.
31. Zheng X, Fan L, Zhou P, Ma H, Huang S, Yu D, Zhao L, Yang S, Liu J, Huang A, et al: Detection of circulating tumor cells and circulating tumor microemboli in gastric cancer. Transl Oncol 10: 431-441, 2017.

32. Galanzha EI, Shashkov EV, Kelly T, Kim JW, Yang L and Zharov VP: In vivo magnetic enrichment and multiplex photoacoustic detection of circulating tumour cells. Nat Nanotechnol 4: 855-860, 2009

33. Zhang H, Jia Z, Wu C, Zang L, Yang G, Chen Z and Tang B: In vivo capture of circulating tumor cells based on transfusion with a vein indwelling needle. ACS Appl Mater Interfaces 7: 20477-20484, 2015.

34. Plaks V, Koopman CD and Werb Z: Cancer. Circulating tumor cells. Science 341: 1186-1188, 2013.

(i) (3) This work is licensed under a Creative Commons

EY No NO Attribution-NonCommercial-NoDerivatives 4.0 International (CC BY-NC-ND 4.0) License. 